\title{
Validação de tecnologia educacional para implementar um programa comunitário na saúde pública
}

\author{
Validation of educational technology to implement a community program in \\ public health
}

\section{AUTORES \\ Lisandra Maria Konrad ${ }^{1}$ (D) \\ Cezar Grontowski Ribeiro ${ }^{1,2}$ (iD \\ Camila Tomicki ${ }^{1}$ iD \\ Tânia Rosane Bertoldo Benedetti ${ }^{1}$ (D) \\ 1 Universidade Federal de Santa Catarina, \\ Programa de Pós-Graduação em Educação Física. \\ Florianópolis, Santa Catarina, Brasil. \\ 2 Instituto Federal do Paraná, Departamento de Educação Física. Palmas, Paraná, Brasil.}

\section{CONTATO}

Lisandra Maria Konrad

lisandrakonrad@hotmail.com

Universidade Federal de Santa Catarina.

Centro de Desportos, Campus Reitor João

David Ferreira Lima, s/n - Trindade. Flori-

anópolis, Santa Catarina, Brasil.

CEP: 88040-900.

DOI

$10.12820 /$ rbafs. $25 \mathrm{e} 0155$

\section{(cc) BY}

Este trabalho está licenciado com uma Licença

Creative Commons - Atribuição 4.0 Internacional.

\begin{abstract}
RESUMO
O objetivo do estudo foi validar o treinamento on-line para implementar o Programa Vida Ativa Melhorando a Saúde "VAMOS", versão 3.0, na Atenção Primária à Saúde. Participaram 48 profissionais (especialistas $=22$; profissionais da saúde $=26$ ) que utilizaram o Suitability Assessment of Materials - SAM para avaliar a adequação do material e o System Usability Scale - SUS para a usabilidade do treinamento. As análises incluíram o Índice de Validade de Conteúdo (IVC $\geq 0,75$ ) e Coeficiente $\operatorname{Kappa}(k=\geq 0,61)$. A adequação do material $(\mathrm{SAM}=92,92 \pm 4,60 ; 95,59 \pm 4,59)$ e a usabilidade (SUS $=96,14 \pm 5,83 ; 97,40 \pm 3,24$ ) apresentaram escores gerais altos para os dois grupos de avaliadores. O IVC $(>0,80)$ e a consistência interna $(k=0,60$ a 0,77$)$ mostraram percentuais de concordância entre 70 e 84\%. Assim, o treinamento on-line foi validado para capacitar profissionais da saúde para implementação do Programa VAMOS.
\end{abstract}

Palavras-chave: Capacitação em serviço; Ciência da implementação; Profissional da saúde; Saúde pública; Tecnologia em saúde.

\section{ABSTRACT}

The present study aims to validate online training to implement Active Life Improving Health Program "VAMOS" version 3.0, in Primary Health Care. A group of 48 professionals (experts $=22$; health professionals $=26)$ used the Suitability Assessment of Materials (SAM) to evaluate the suitability of the material and the System Usability Scale (SUS) for online usability. The analyzes included the Content Validity Index (CVI $\geq 0.75)$ and Kappa Coefficient $(k=\geq 0.61)$. The suitability of the material ( $S A M=92.92 \pm 4.60 ; 95.59 \pm 4.59)$ and the usability ( $S U S=96.14 \pm 5.83 ; 97.40 \pm$ $3.24)$ presented high overall scores for the two groups of evaluators. The CVI $(>0.80)$ and internal consistency $(k=0.60$ to 0.77$)$ showed agreement percentages between 70 and $84 \%$. Thus, the online training was validated to train health professionals to implement VAMOS Program.

Keywords: Inservice training; Implementation science; Health personnel; Public health; Biomedical technology.

\section{Introdução}

Uma vez estabelecida a preocupação mundial com as doenças crônicas não transmissíveis (DCNTs) as políticas públicas estão voltadas para a implementação de estratégias de mudança no estilo de vida da população' ${ }^{1}$. No Brasil, a maioria destas ações integram um conjunto de atividades educativas desenvolvidas na Atenção Primária à Saúde (APS) direcionadas a promoção da atividade física e alimentação saudável na comunida$\mathrm{de}^{2}$, ainda que com uma baixa adesão ${ }^{3}$.

Reconhecidamente, a gestão do cuidado em saúde é baseada em evidências clínicas que fornecem diretrizes sobre o que deve ser aplicado na prática. Mas, apesar do esforço empreendido no planejamento, as informações e os recursos disponíveis para os tomadores de decisão de como implementar programas comunitários são incipientes ${ }^{4}$.

Neste sentido, um protocolo de base comunitária Programa Vida Ativa Melhorando a Saúde "VAMOS”, vem sendo testado no âmbito da APS. Ele foi desenvolvido para ser viável para populações de qualquer nível de instrução e de diferentes regiões brasileiras, podendo ser adaptado de acordo com a realidade cultural, social e econômica ${ }^{5}$. A iniciativa reúne evidências de efetividade na saúde pública ${ }^{6}$, contudo a retenção atinge em torno de $50 \%$ dos participantes ${ }^{7}$, justificando 
assim, a necessidade de aprimorar o processo de disseminação e facilitar a implementação da intervenção. Neste sentido, foi desenvolvido um treinamento on-line para capacitar profissionais da saúde ${ }^{8}$

A utilização de ambientes virtuais de aprendizagem vem subsidiando os procedimentos educativos nos cuidados em saúde com resultados eficazes na resolução de problemas. Além do alcance e do potencial no processo de aprendizagem, servem como ferramentas de apoio organizacional na promoção da saúde ${ }^{9}$. Contudo, antes de serem disseminados, estes recursos devem ser avaliados para determinar em que grau comunicam informações de saúde de maneira clara e eficaz ao adaptar intervenções. Desta forma, o objetivo deste estudo foi validar a adequação do material e a usabilidade do treinamento on-line para capacitar profissionais da saúde para implementação do Programa VAMOS, versão 3.0, na APS.

\section{Métodos}

Trata-se de um estudo metodológico realizado a partir do projeto de intervenção de base comunitária: "Programa VAMOS: do treinamento a implementação". Foi aprovado pelo Comitê de Ética em Pesquisa com Seres Humanos da Universidade Federal de Santa Catarina (sob no 1.360.210) e pode ser encontrado no Registro Brasileiro de Ensaios Clínicos (http://www. ensaiosclinicos.gov.br/) pelo indicador RBR-2vw77q.

O VAMOS é um programa de mudança de comportamento que objetiva motivar adultos e idosos a adotarem um estilo de vida ativo e saudável em relação à atividade física e a alimentação ${ }^{5}$. $\mathrm{Na}$ versão atual (3.0), a modalidade presencial foi desenvolvida para ser implementada durante nove meses em encontros (semanais, quinzenais e mensais) de $1 \mathrm{~h} 30$ min a 2 horas de duração conduzidos por um profissional da saúde. O material didático das 18 seções do programa aborda temas relacionados aos benefícios da atividade física e da alimentação saudável, além do desenvolvimento de estratégias para efetuar e manter as mudanças necessárias. Para garantir a efetividade do protocolo foi desenvolvida uma ferramenta educacional para auxiliar na implementação. Trata-se de um treinamento on-line oferecido, gratuitamente, na modalidade de Ensino a Distância (EaD) com objetivo de capacitar profissionais da saúde (multiplicadores) para planejar, conduzir e avaliar o programa em diferentes contextos ${ }^{8}$. Para atender ao novo protocolo do VAMOS versão 3.0, o treinamento on-line passou por duas etapas de reestruturação.

A primeira etapa correspondeu a análise e atualização do conteúdo. A nova matriz de conteúdo foi elaborada a partir da leitura das 12 seções do treinamento anterior ${ }^{8}$, atualizando informações e ajustando temas e objetivos. Este processo foi conduzido por dois pesquisadores individualmente e, em seguida, um terceiro pesquisador fez uma avaliação final. A etapa foi concluída após revisão do material por um especialista da área de Letras.

O modelo atual (Quadro 1) apresenta cinco módulos que contemplam temas, objetivos e conteúdos divididos em 13 unidades. $\mathrm{O}$ material apresenta uma sequência de conteúdo para que o cursista conheça a proposta do programa (Módulo I), os conceitos e marcadores para mudança de comportamento (Módulo II), as estratégias para gerenciar grupos de pessoas (Módulo III), a estrutura e os conteúdos para implementação (Módulo IV) e, por fim, as formas de avaliação do programa (Módulo V).

A segunda etapa correspondeu a análise do layout e design instrucional. $\mathrm{O}$ treinamento é ambientado na

Quadro 1 - Matriz da estrutura e conteúdo do treinamento on-line para implementação do Programa VAMOS, versão 3.0, 2019.

\begin{tabular}{|c|c|c|c|}
\hline Módulo & Unidade & Conteúdo & Indicador \\
\hline I. Conhecendo o VAMOS & $\begin{array}{l}\text { Programa VAMOS } \\
\text { Base teórica do VAMOS }\end{array}$ & $\begin{array}{l}\text { Objetivo, missão, filosofia do programa e constructos teóricos } \\
\text { que fundamentam a mudança de comportamento. }\end{array}$ & Proposta \\
\hline $\begin{array}{l}\text { II. Mudando o estilo de } \\
\text { vida com o VAMOS }\end{array}$ & $\begin{array}{l}\text { Estilo de vida } \\
\text { Atividade física } \\
\text { Alimentação saudável }\end{array}$ & $\begin{array}{l}\text { Conceitos e diretrizes para adotar um estilo de vida ativo } \\
\text { e saudável considerando os desfechos da atividade física e } \\
\text { alimentação. }\end{array}$ & Marcadores \\
\hline $\begin{array}{l}\text { III. Conduzindo grupos de } \\
\text { pessoas no VAMOS }\end{array}$ & $\begin{array}{l}\text { Princípios e estilos } \\
\text { de aprendizagem } \\
\text { Tipos de comportamentos } \\
\text { Administração do estresse } \\
\text { Estratégias de motivação }\end{array}$ & $\begin{array}{l}\text { Estratégias para gerenciar e motivar a mudança de } \\
\text { comportamento de um grupo de pessoas. }\end{array}$ & Estratégias \\
\hline IV. Implantando o VAMOS & $\begin{array}{l}\text { Conteúdo do VAMOS } \\
\text { Estrutura do VAMOS } \\
\text { Adaptações no VAMOS }\end{array}$ & Seções, temas e a estrutura para conduzir o programa. & Implementação \\
\hline V. Avaliando o VAMOS & $\begin{array}{l}\text { Avaliação do processo e dos resultados } \\
\text { do VAMOS }\end{array}$ & Formas de monitorar e estimar a efetividade do programa. & Avaliação \\
\hline
\end{tabular}


Plataforma Moodle (Modular Object Oriented Dynamic Learning Environment) e hospedado na Superintendência de Governança Eletrônica e Tecnologia da Informação e Comunicação da Universidade Federal de Santa Catarina. A matriz do design instrucional foi elaborada com elementos de comunicação visual para oferecer um ambiente responsivo, com linguagem amigável para facilitar a leitura e a realização das atividades. Três especialistas da área do design do Laboratório de Orientação da Gênese Organizacional (LOGO) da referida instituição criaram a diagramação, adequando a fonte, disposição de texto, paleta de cores, ilustrações, imagens e demais elementos gráficos.

O novo formato da plataforma on-line disponibiliza um menu com sequência instrucional que oferece na página inicial acesso a apresentação com o objetivo do treinamento e cinco módulos com os temas abordados (acesse layout da plataforma em: https://cutt.ly/egRsKqr). Cada módulo contém unidades com uma estrutura (introdução, objetivo, conteúdo, síntese e referências) que norteia a trilha de aprendizagem. Após a leitura de cada unidade o cursista é conduzido às atividades de fixação (cinco questões objetivas de múltipla escolha) e, ao finalizar o treinamento, à uma avaliação com 30 questões objetivas de múltipla escolha. A certificação é fornecida com desempenho de $80 \%$ nas respostas.

A nova versão do treinamento foi avaliada por um painel de experts da área acadêmica (especialistas) e do serviço de saúde (público alvo). O recrutamento dos especialistas seguiu três critérios de inclusão: atuar no ensino superior, possuir conhecimento nas áreas que envolveram a elaboração do treinamento on-line (Educação Física, Nutrição, Psicologia, Design) e ter titulação mínima de especialista. Para o público alvo os critérios estabelecidos foram: atuar na APS e ter concluído o treinamento on-line.

Para avaliar a adequação do material foi utilizado o Suitability Assessment of Materials (SAM) que considera seis itens: conteúdo, linguagem, ilustrações gráficas, layout e design, motivação para o aprendizado e adequação cultura ${ }^{10}$. O ambiente virtual foi avaliado pelo System Usability Scale (SUS) que determina a usabilidade por meio de cinco componentes: facilidade de aprendizagem, facilidade de memorização, identificação de inconsistências, eficiência e satisfação dos usuários $^{11}$. Os itens dos instrumentos são avaliados por uma escala tipo Likert para o material $(0=$ inadequado; 1 = parcialmente adequado; 2 = adequado) e para usabilidade $(1$ = discordo totalmente; 2 = discordo; 3 = não concordo, nem discordo; 4 = concordo 5 = concordo totalmente), gerando uma pontuação que indica boa adequação do material $(>60)^{12}$ e usabilidade $(>70)^{13}$.

Os especialistas foram convidados por meio eletrônico e receberam acesso (chave e senha) a plataforma e aos links com os instrumentos de avaliação (formulários do Google Docs). O público alvo teve acesso ao treinamento por meio da inscrição na plataforma on-line aberta nos anos de 2018 e 2019 e avaliaram o treinamento com os mesmos instrumentos direcionados aos especialistas. Ambos os grupos realizaram a leitura de todos os elementos (temas, objetivos, conteúdo e atividades) divididos nas 13 unidades dos cinco módulos do treinamento. Todos os avaliadores leram e assinaram o Termo de Consentimento Livre e Esclarecido.

Foram realizados cálculos por meio do Índice de Validade de Conteúdo (IVC) utilizando a concordância dos avaliadores quanto à representatividade dos itens (critério $=\mathrm{IVC} \geq 0,75$ ). A consistência interna foi avaliada pelo Coeficiente Kappa de Fleiss ( $k$ ) gerando o percentual de concordância $(\% \mathrm{C})$ entre os avaliadores (critério $=\% \mathrm{C} \geq 0,61$ ). Os dados foram analisados no software $\mathrm{SPSS}^{\circledR} 22.0$ for Windows.

\section{Resultados}

A taxa de retorno dos avaliadores foi de 73,3\% entre os especialistas e $41,5 \%$ entre os profissionais da saúde. Desta forma, participaram do painel de especialistas 22 profissionais vinculados à instituições públicas brasileiras, que tinham média de idade de 35,23 anos $( \pm 6,38)$, em sua maioria mulheres $(77,3 \% ; \mathrm{n}=17)$, com titulação de doutorado $(36,4 \% ; \mathrm{n}=8)$, mestrado $(31,8 \%$; $\mathrm{n}$ $=7)$ e especialização $(31,8 \% ; n=7)$ atuando em média 10,91 anos $( \pm 5,90)$ nas áreas da Educação Física, Nutrição, Psicologia e Design.

O público alvo foi composto de 26 profissionais da saúde, com média de idade de 37,04 anos $( \pm 7,77)$, em sua maioria mulheres $(88,5 \%$; $=23)$ com pós-graduação $(65,4 \% ; n=17)$, atuando em média 6,42 anos $( \pm 5,56)$ na APS nas áreas da Educação Física, Enfermagem, Nutrição, Psicologia, Serviço Social, Farmácia, Fisioterapia e Medicina.

$\mathrm{Na}$ avaliação da adequação do material, o escore geral do SAM foi considerado adequado para os dois grupos de avaliadores $(\mathrm{SAM}>90)$. O item "ilustrações gráficas" apresentou o menor escore $(\mathrm{SAM}=78,44)$ entre os especialistas refletindo em índices de concordância abaixo do esperado (IVC $=0,61 ; k=0,24 ; \% \mathrm{C}$ = 49,11). Por outro lado, na avaliação do público alvo o 
valor do escore foi elevado para o item $(\mathrm{SAM}=94,23)$ e demais índices (IVC $=0,88 ; k=0,69)$ apresentando uma concordância dentro do critério estabelecido (\%C $=79,43)$ - Tabela 1 .

Os escores gerais da usabilidade mostraram alta concordância para ambos os grupos de avaliadores (SUS > 95) refletindo valores aceitáveis de IVC $(>0,75)$. Ainda que para os especialistas os valores do Kappa tenham sido mais baixos para os itens de "facilidade de memorização", "identificação de inconsistências" e "eficiência”, o percentual de concordância apresentou valores aceitáveis $(\% \mathrm{C}>0,61)$ validando os itens (Tabela 1$)$.

\section{Discussão}

Os parâmetros avaliados apresentaram valores acima dos critérios estabelecidos para a maioria dos itens, demonstrando consistência nas respostas e concordância entre os avaliadores. Quando da elaboração de materiais educativos em saúde, pesquisadores sugerem como essencial o uso de instrumentos que possam responder a construção de tecnologias que contenham conteúdo específico, estrutura coerente e linguagem apropriada para facilitar a aprendizagem ${ }^{14}$.
O SAM oferece um método objetivo para avaliar a adequação das informações em saúde por meio de itens que devem estar alinhados com o propósito do material desenvolvido e ao público destinado ${ }^{10}$. Observou-se neste estudo que o material foi considerado adequado ao objetivo do treinamento, com exceção apenas do item "ilustrações gráficas" quando avaliado pelo painel de especialistas. Este item diz respeito às imagens inseridas no material cujo papel é auxiliar na compreensão do conteúdo ao longo do texto. $\mathrm{Na}$ avaliação do público alvo algumas imagens já haviam sido substituídas para adequar o material à avaliação dos especialistas, o que pode justificar a diferença nos percentuais de adequação entre ambos os grupos de avaliadores.

O uso das inovações tecnológicas na área da saúde facilita o processo de trabalho de equipes multiprofissionais, qualificando a assistência e aumentando a resolutividade no cuidado9. Contudo, reorientar as condutas para promoção da saúde e implementar ações tem sido um desafio, uma vez que envolve fatores relacionados não somente a atributos pessoais, mas, ao contexto da organização e aos recursos disponíveis ${ }^{4,9}$. Assim, para ampliar o acesso a capacitações que im-

Tabela 1 - Avaliação da adequação do material e usabilidade do treinamento on-line para implementação do Programa VAMOS, versão 3.0, Florianópolis, Brasil, 2019 ( $\mathrm{n}=48$ ).

\begin{tabular}{|c|c|c|c|c|c|c|c|c|}
\hline & \multicolumn{4}{|c|}{ Especialistas } & \multicolumn{3}{|c|}{ Público Alvo } & \\
\hline & \multicolumn{4}{|c|}{$\mathrm{n}=22$} & \multicolumn{3}{|c|}{$\mathrm{n}=26$} & \\
\hline \multicolumn{9}{|l|}{ Adequação do Material } \\
\hline Itens $\mathrm{SAM}^{*}$ & Escore & $\mathrm{IVC}^{* * * *}$ & $\mathrm{k}^{* * * * *}$ & $\% \mathrm{C}^{* * * * * *}$ & Escore & IVC & $\mathrm{k}$ & $\% \mathrm{C}$ \\
\hline Conteúdo & 97,73 & 0,97 & 0,87 & 91,14 & 99,04 & 0,98 & 0,94 & 96,19 \\
\hline Linguagem & 97,73 & 0,95 & 0,87 & 91,24 & 97,28 & 0,94 & 0,78 & 85,44 \\
\hline Ilustrações gráficas & 78,44 & 0,61 & 0,24 & 49,11 & 94,23 & 0,88 & 0,69 & 79,43 \\
\hline Layout e design & 96,21 & 0,88 & 0,68 & 78,37 & 96,15 & 0,92 & 0,78 & 85,61 \\
\hline Motivação & 97,97 & 0,94 & 0,83 & 88,44 & 95,15 & 0,91 & 0,75 & 83,45 \\
\hline Adequação cultural & 95,45 & 0,91 & 0,75 & 83,09 & 87,50 & 0,88 & 0,69 & 79,19 \\
\hline Média & 92,92 & 0,88 & 0,71 & 80,23 & 95,69 & 0,92 & 0,77 & 84,89 \\
\hline Desvio Padrão & $\pm 4,60$ & $\pm 0,13$ & $\pm 0,24$ & $\pm 16,05$ & $\pm 4,59$ & $\pm 0,04$ & $\pm 0,09$ & $\pm 6,21$ \\
\hline \multicolumn{9}{|c|}{ Usabilidade } \\
\hline Itens SUS** & Escore & IVC & $\mathrm{k}$ & $\% \mathrm{C}$ & Escore & IVC & $\mathrm{k}$ & $\% \mathrm{C}$ \\
\hline Facilidade de aprendizagem & 97,16 & 0,88 & 0,70 & 77,87 & 97,36 & 0,89 & 0,75 & 80,90 \\
\hline Facilidade de memorização & 94,32 & 0,77 & 0,51 & 63,20 & 98,08 & 0,92 & 0,80 & 85,23 \\
\hline Inconsistências & 95,45 & 0,82 & 0,58 & 68,83 & 97,12 & 0,85 & 0,64 & 72,92 \\
\hline Eficiência & 94,70 & 0,77 & 0,52 & 64,34 & 97,76 & 0,88 & 0,72 & 79,32 \\
\hline Satisfação & 97,35 & 0,88 & 0,71 & 78,37 & 97,12 & 0,87 & 0,70 & 77,36 \\
\hline Média & 96,14 & 0,82 & 0,60 & 70,52 & 97,50 & 0,88 & 0,72 & 79,15 \\
\hline Desvio Padrão & $\pm 5,83$ & $\pm 0,06$ & $\pm 0,10$ & $\pm 7,25$ & $\pm 3,24$ & $\pm 0,03$ & $\pm 0,06$ & $\pm 4,53$ \\
\hline
\end{tabular}

*SAM $=$ Suitability assessment of materials $; *$ SUS = System usability scale $; * *$ IVC $=$ índice de validade de conteúdo (calculado considerando o número de respostas "adequado" para SAM e "concordo totalmente" para o SUS dividido pelo número total de respostas para cada item); ${ }^{* * * * k}$ = Coeficiente de kappa de fleiss; ${ }^{* * * * * \%} \% \mathrm{C}=$ percentual de concordância. 
pactem neste processo, além da construção de materiais didáticos que sintetizem as informações, é necessário construir plataformas de fácil uso ${ }^{15}$.

O SUS tem sido utilizado para avaliar a usabilidade percebida de usuários, produtos e serviços na área da saúde ${ }^{15}$. Os resultados do SUS neste estudo demostraram um ambiente virtual favorável a aprendizagem e com alto nível de satisfação pelos avaliadores. A ciência da implementação usa de estratégias para adaptar intervenções para o mundo real, considerando a evidência científica e o contexto como elementos que influenciam no sucesso da implementação e sustentabilidade nas ações de saúde pública ${ }^{16}$. Mas, essa transição é de natureza complexa e influenciada por inconsistências entre os objetivos daqueles que desejam implementar novas estratégias e os membros da comunidade que devem assumir novos comportamentos ${ }^{16}$.

Assim, a adoção de práticas que combinam evidências com recursos educacionais administrados em ambiente virtual, pode acelerar a disseminação de programas de promoção da saúde, uma vez que apresentam rápida transmissão das informações e proporcionam amplo acesso. Além disso, estes sistemas podem facilitar a transferência das diretrizes para a prática clínica, ampliar a implementação de ações na saúde e ter um impacto positivo no cuidado primário da população.

Ainda que não tenha sido realizada uma análise qualitativa, a metodologia usada foi vantajosa, uma vez que o consenso atribuído aos itens analisados contribuiu para um treinamento confiável e coerente, tendo em vista a experiência e qualificação dos avaliadores. Outro ponto relevante é que, muito embora esta ferramenta tenha sido direcionada a um protocolo específico de intervenção, poderá servir como modelo para planejar outras ações de promoção da saúde.

Com base no exposto, concluiu-se que o treinamento on-line apresentou validade de conteúdo e usabilidade para capacitar profissionais da saúde para a implementação do Programa VAMOS, versão 3.0, na APS. Tendo em vista a importância do recurso educacional na qualificação profissional, o estudo contribui para a prática clínica por tratar-se de uma tecnologia inovadora alinhada às políticas públicas e capaz de facilitar a disseminação e a implementação de intervenções de promoção da saúde no âmbito da saúde pública.

\section{Conflito de interesses}

Os autores declaram não haver conflito de interesses.

\section{Financiamento}

O estudo recebeu financiamento por meio do edital FAPESC No 10/2015-MS-DECIT/CNPq/SES-SC - PPSUS, no 484/2016, no Termo de Outorga: 2016TR2210.

\section{Contribuição dos autores}

Konrad LM, participou da concepção inicial do estudo, coleta, análise e interpretação dos dados, redação e revisão crítica do texto. Ribeiro CG e Tomicki C, contribuíram para a redação do artigo e revisão crítica do conteúdo intelectual. Benedetti TRB, foi responsável pelo envio do projeto e obtenção do financiamento, pela revisão crítica do artigo e aprovação da versão final.

\section{Agradecimentos}

Os autores agradecem ao Laboratório de Orientação da Gênese Organizacional (LOGO) e da Superintendência de Governança Eletrônica e Tecnologia da Informação e Comunicação da Universidade Federal de Santa Catarina pelo apoio na elaboração da nova edição do treinamento on-line. Aos especialistas das instituições de ensino superior e aos profissionais do serviço da saúde que aceitaram avaliar o treinamento.

\section{Referências}

1. World Health Organization. Noncommunicable diseases progress monitor 2017 [Internet]. [citado em 2019 Dez 17] Disponível em: http://www.who.int/nmh/publications/en/.

2. Wolker SM, Sandreschi OS, Tomicki C, Konrad LM, Quadros NE, Ribeiro CG, et al. Monitoring the health academy program from 2015 to 2017. Rev Andal Med Deporte. 2020;13(1):16-20.

3. Ferreira RW, Caputo EL, Häfele CA, Jerônimo JS, Florindo AA, Knuth AG et al. Acesso aos programas públicos de atividade física no Brasil: Pesquisa Nacional de Saúde, 2013. Cad Saúde Pública. 2019;35(2):e00008618.

4. Schneider LR, Pereira RPG, Ferraz L. A prática baseada em evidência no contexto da Atenção Primária à Saúde. Saúde Debate. 2018;42(118):594-605.

5. Benedetti TRB, Manta SW, Gomez LSR, Rech CR. Logical model of a behavior change program for community intervention - Active Life Improving Health - VAMOS. Rev Bras Ativ Fís Saúde. 2017; 22(3):309-13.

6. Meurer ST, Lopes ACS, Almeida FA, Mendonça RD, Benedetti TRB. Effectiveness of the VAMOS strategy for increasing physical activity and healthy dietary habits: a randomized controlled community trial. Health Educ Behav. 2019;46(3):406-16.

7. Konrad LM, Tomicki C, Ribeiro CG, Bezerra JB, Maciel EC, Rech CR, et al. Length of stay in a behavior change program in primary health care: "VAMOS” Program. Rev Bras Ativ Fis Saúde. 2019;24:e0090.

8. José HPM, Konrad LM, Ribeiro CG, Benedetti, TRB. Validação do treinamento online para multiplicadores do Programa Vida Ativa melhorando a Saúde (VAMOS).J Phys Educ. 2019;30:e3040.

9. Santos AF, Fonseca DS, Araújo LL, Procópio CSD, Lopes EAS, Lima AMLD, et al. Incorporação de tecnologias de informação e comunicação e qualidade na atenção básica em saúde no Brasil. Cad Saúde Pública. 2017;33(5):e00172815. 
10. Souza CS, Turrini RNT, Poveda VB. Tradução e adaptação do instrumento "Suitability Assessment of Materials"(SAM) para o português. Rev Enferm UFPE on line. 2015;9(5):7854-61.

11. Tenório JM, Cohrs FM, Sdepanian VL, Pisa IT, Marin HF. Desenvolvimento e avaliação de um protocolo eletrônico para atendimento e monitoramento do paciente com doença celíaca. RITA. 2010;17(2):211-220.13.

12. Doak C, Doak L, Root J. Assessing suitability of materials. In: Teaching patients with low literacy skills (2 Ed.). Philadelphia (PA): J.B.Lippincott. 1996; 4 chapter:41-60.

13. Bangor A, Kortum PT, Miller JT. An empirical evaluation of the System Usability Scale. Int J Hum-Comput Int. 2008:24(6):574-94.
14. Leite SS, Áfio ACE, Carvalho LV, Silva JM, Almeida PC, Pagliuca LMF. Construction and validation of an educational content validation instrument in health. Rev Bras Enferm. 2018;71(Suppl 4):1635-41.

15. Lewis JR. The System Usability Scale: past, present, and future. Int J Hum-Comput Int. 2018;34(7):577-90.

16. Mathieson A, Grande G, Luker K. Strategies, facilitators and barriers to implementation of evidence-based practice in community nursing: a systematic mixed-studies review and qualitative synthesis. Prim Health Care Res Dev. 2018;20(e6):1-11.

\section{Como citar este artigo:}

Konrad LM, Ribeiro CG, Tomicki C, Benedetti TRB. Validação de tecnologia educacional para implementar um programa comunitário na saúde pública. Rev Bras Ativ Fis Saúde. 2020;25:e0155. DOI: 10.12820/rbafs.25e0155

Recebido: 07/05/2020

Aprovado: 03/11/2020 